\title{
Indicadores de enfermedades mentales en pacientes mexicanos con VIH/SIDA y su relación con la adherencia terapéutica
}

\author{
Ismael Garcia-Cedillo \\ Universidad Autónoma de San Luis Potosí, México \\ Dirección postal: Cordillera Karakorum 536, Col. Lomas, $3^{\text {a }}$. Sección, CP 78216, SLP, México. \\ Ce: ismaelgace@yahoo.com.mx
}

Fabiola Alfaro-Castro Mónica Rodriguez-Delgado Omar Sánchez-Armáss Cappello

Universidad Autónoma San Luis Potosí, México.

Resumen. Objetivo. Se analiza la relación entre algunos indicadores de enfermedades mentales y la adherencia terapéutica en pacientes con VIH/SIDA. Participantes. Muestra de 76 pacientes mexicanos infectados por VIH/SIDA. Procedimiento. Entrevista a profundidad a pacientes de un hospital público de la ciudad de San Luis Potosí, México. Resultados. 80\% de los participantes presentó indicadores de enfermedades mentales correspondientes a: $28 \%$ conducta antisocial; $20 \%$ depresión clínica; $13 \%$ síndrome de estrés postraumático, 12\% psicosis orgánica y $8 \%$ discapacidad intelectual. El porcentaje de pacientes infectados con VIH/SIDA con indicadores de enfermedad mental concuerda con los resultados de investigaciones realizadas en otros países. Solamente $7 \%$ de los participantes mostró un nivel de adherencia apropiado. La adherencia no guarda una relación lineal con la presencia de indicadores de enfermedad mental. La adherencia se relaciona con la escolaridad, la utilización de estrategias para recordar la toma del medicamento, confiar el estatus a los familiares y apoyo familiar.

Palabras clave: adherencia terapéutica, enfermedad mental, VIH/SIDA.

Abstract. Objective: This study analyzes the relation between mental illness indicators and therapeutic adherence in people with HIV/AIDs. Participants: 76 Mexican patients infected with HIV/AIDs. Procedure: In-depth interview to the participants in a public hospital in San Luis Potosí, Mexico. Results: $80 \%$ of the sample presented indicators of mental illness: $28 \%$ antisocial behavior, 20\% clinical depression, 13\% Posttraumatic Stress Disorder, 12\% organic psychosis and $8 \%$ intellectual disability. These results are similar to findings reported in other countries. Only $7 \%$ of the participants get a high level of therapeutic adherence. The adherence has a complex non-linear relation with the mental health status, and is related to education, the use of some strategy in order to remember medication intake, the possibility of revealing their status to family, and family support.

Key Words: Therapeutic adherence, mental illness, HIV/AIDs. 


\section{Introducción}

La adherencia terapéutica resulta muy importante si queremos lograr la estabilización de la salud de los pacientes con VIH/SIDA. Los médicos recomiendan una adherencia casi perfecta (al menos del 95\%), para lograr una mejora importante en la salud del paciente (Ingersoll, 2004; Horne, Buick, Fisher, Leake, Cooper y Weinman, 2004). Sin embargo, ser adherente resulta un verdadero reto para las personas con VIH/SIDA ya que los regímenes suelen ser complejos para pacientes poco acostumbrados a la sistematización en la toma de medicamentos y pueden producir efectos secundarios desagradables (Johnson, Chesney, Goldstein, Remien, Catz, Gore-Felton, Charlebois, y Morin, 2006), además de que muchos pacientes prefieren mantener la confidencialidad con respecto a su estatus y por tanto únicamente toman su medicina cuando están a solas. Por lo anterior, de acuerdo con Demmer (2003) y Chesney (2003), el logro de un óptimo nivel de adherencia terapéutica requiere de varias condiciones, como información pertinente y objetiva acerca de la enfermedad, apoyo familiar y de amistades, un sistema de salud que tome en cuenta su idiosincrasia, etc. Además, se necesita que los pacientes alcancen un buen nivel de salud mental (Hill, Kendall y Fernández, 2003).

En el presente estudio se evaluaron mediante entrevistas a profundidad algunos indicadores de enfermedad mental de una muestra de 76 pacientes infectados con el VIH/SIDA y se identificó la relación entre estos indicadores y la adherencia terapéutica.

Con la introducción de los antirretrovirales en la terapia HAART (Highly Aggressive Anti-Retroviral Treatment) ha cambiado radicalmente el curso clínico de la infección por VIH, pues puede devenir de una enfermedad incurable a una enfermedad crónica, pero se requiere de un alto nivel de adherencia al tratamiento (Balfour, Silvermani, Tasca, Angelli, Macpherson, Garber, Cooper y Cameron, 2006). La no adherencia puede ocasionar un rebrote viral, situación desalentadora porque si el virus en una persona se vuelve resistente, entonces el tratamiento se torna ineficaz y además, si la persona desarrolla cepas resistentes a los fármacos, puede transmitir el virus resistente a otras personas a través del contacto sin protección (Paredes-Castellanos, 2002). Entre menos adherencia terapéutica exista, mayores serán las posibilidades de falla virológica y deterioro del paciente (Carrillo-Martínez, García-Lozada y Sierra-Corona, 2005).

Considerando la situación mencionada, los médicos recomiendan un 95\% de adherencia para lograr una mejora importante en la salud del paciente (Chesney, 2003; Weiss, French, Finkelstein, Waters, Mukherjee y Agins, 2003; Ciasullo y Escovitz, 2005). La adherencia al tratamiento es esencial para reducir la mortalidad y la morbilidad ente los pacientes viviendo 
con VIH/SIDA (Uldall, Palmer, Whetten y Mellins, 2005; Reback, Larkins y Shoptaw, 2003).

La adherencia terapéutica consiste en el apego del paciente al régimen prescrito por su médico, pero no resulta fácil de definir, pues no solo está compuesta por las actitudes y conductas del paciente con respecto al tratamiento (que es a lo que comúnmente se le ha reducido), sino que es una condición multivariable (Broyles y Erlen, 2005), además de que no existe un solo factor al que se pueda atribuir el no apego al tratamiento (TorresEscobar, González-López, y Martínez-Fernández, 2002).

Para definir la adherencia se tiene que tomar el intervalo de tiempo que se quiere considerar y sus indicadores, esto es, si los pacientes acuden con regularidad a sus citas médicas, si se realizan los análisis pertinentes, si toman sus medicamentos de la manera prescrita y si adoptan conductas más saludables. Incluso, se sabe que el simple paso del tiempo hace más difícil la adherencia. Carrieri, Chesney, Spire, Loundou, Sobel, Lepeu, Moatti y el MANIF Study Group (2003), señalan que recientemente se ha mostrado que la adherencia al HAART varía a lo largo del tiempo. En su estudio se encontró que un cuarto de los pacientes que eran inicialmente adherentes al HAART declinó en un período de 18 meses.

Otra cuestión que hace más compleja la definición de la adherencia terapéutica, de acuerdo con Johnston y Mann (2003), es que la no adherencia se puede clasificar como intencional y no-intencional. Por ejemplo, el olvido algunas veces de la ingesta de medicamentos es no-intencional. Como señalan estos autores, hasta ahora no se ha estudiado a fondo la noadherencia intencional, esto es, cuando el paciente se da "vacaciones" o cuando deja de ingerir el medicamento algún fin de semana para poder usar alcohol o drogas o simplemente para "descansar" de los fármacos. La decisión de adherirse o no al tratamiento nunca es final, sino que el paciente constantemente la está negociando consigo mismo. De acuerdo con dichos autores, entre sus determinantes están las circunstancias particulares y creencias religioso-espirituales, el estado de salud y la interacción con otras personas.

A pesar de la enorme importancia de la adherencia terapéutica para el logro no solamente de la supervivencia, sino también de una buena calidad de vida para los pacientes infectados con el VIH/SIDA, ser adherente resulta un verdadero reto, por la complejidad de los regímenes y por los efectos secundarios (Johnson, et al., 2006). Así pues, López-Martínez, OrtízMondragón, Moreno-Monroy, Sierra-Corona, Morales-Carcaño, MagisRodríguez, Hernández-Tepichín y Baez-Villaseñor (1999) realizaron un estudio en el que no encontraron un solo paciente mexicano con un 100\% de adherencia. Los pacientes que participaron en su estudio señalaron como factor principal de no adherencia a la estigmatización tanto laboral, social como familiar. 
Con respecto a la salud mental, son muchos los estudios que reportan la presencia de indicadores de enfermedad mental entre los pacientes con VIH/SIDA en el ámbito internacional. Wagner, Kanouse, Koegel y Sullivan (2004), por ejemplo, encontraron que la prevalencia de VIH entre personas que presentan problemas de salud mental es ocho veces mayor a la que presenta la población en general, lo que los llevó a considerar a la enfermedad mental como un factor de riesgo para una adherencia pobre.

Ingersoll (2004) observó que un tercio de los pacientes con VIH/SIDA tenía algún desorden mental, como depresión mayor, algún trastorno de ansiedad y abuso de alcohol, desórdenes que complicaban la adherencia de los pacientes al tratamiento. En su estudio, las variables demográficas no tuvieron valor predictivo para la no-adherencia. Sí la tuvo la presencia de desórdenes mentales, particularmente los desórdenes de ansiedad y el abuso de sustancias, en especial de la cocaína.

Murphy, Marelich, Hoffman y Steers (2004) encontraron que las personas con un menor control conductual y emocional tendían a tener problemas de adherencia. Generalmente, este tipo de características suelen encontrarse en personas con alguna enfermedad mental o algún problema psicoafectivo. En el estudio de Wagner, Kanouse, Koegel y Sullivan (2003), una muestra de personas con enfermedad mental que eran VIH positivas mostró tener la misma capacidad que los demás para ser adherentes. Pese a ello suelen tener una adherencia baja (casi un tercio de estos pacientes -con enfermedad mental y VIH positivos- tenían una pobre adherencia). Sin embargo, el $86 \%$ de estos mismos pacientes reportaron tener una buena adherencia a su medicamento psiquiátrico. Por su parte, Royal, Cohn, Kwait, Kidder, Wolitski, Stall, Aidala y Holtgrave (2006) encontraron que los factores asociados con una mala adherencia son: problemas de acceso al sistema de salud, pobre salud mental, uso de sustancias y una actitud pobre hacia el tratamiento.

La carga emocional que impone el diagnóstico de VIH/SIDA sobre los pacientes es muy alta, por lo que no extraña que desarrollen síntomas del Síndrome de Estrés Post-Traumático (SEPT). Se ha encontrado una alta incidencia del SEPT en la población infectada y algunos estudios también han encontrado una alta incidencia de consumo de sustancias y alcohol (Delahanty, Bogart y Figler, 2004; Brief, Bollinger, Vielhauer, BergerGreenstein, Morgan, Brady, Buondonno y Keane, 2004). Debe tomarse en cuenta que existen resultados mixtos, ya que a veces la presencia del SEPT se relaciona de manera directa con la baja adherencia y, en otras, se relaciona con el consumo de los medicamentos fuera de tiempo. Unos relacionan el SEPT con una alta carga viral y una disminución de las células conocidas como CD4 y otros con una carga viral alta pero igualmente con una alta tasa de CD4. Como se podrá apreciar, los resultados son mixtos y complejos. 
Arendt (2006) menciona que los trastornos afectivos que más comúnmente se presentan en los pacientes que viven con VIH son: desorden bipolar I y II y depresión o episodios depresivos. Ahora bien, es difícil llegar a conclusiones con respecto a la prevalencia e incidencia de desórdenes psiquiátricos. De acuerdo con dicho autor, esto se debe, en parte, a que estudios anteriores han situado a las personas con enfermedades psiquiátricas (en especial las psicosis, la depresión mayor y el desorden por abuso de sustancias) como una población en especial riesgo de ser contagiadas por VIH.

El factor predictor más importante de la adherencia, de acuerdo con Barrios-Nuñez, Solís-Cano y Feregrino (2006) y Schwartz, Wilson, Holman, Weber, Cook y Minkoff (2006) es la depresión. Remor (2002) asocia claramente a la depresión con los niveles de adherencia que desarrollan los pacientes, además de otros factores psicosociales como son la ansiedad, el estrés y el apoyo social. Jones, Ishii, LaPerriere, Stanley, Antoni, Ironson, Schneiderman, Van Splunteren, Cassells, Alexander, Gousse, Vaughn, Brondolo, Tobin y Weiss (2003), confirman este hallazgo al asociar la no adherencia con la depresión, la ansiedad y las autoacusaciones en los pacientes. Mellins, Kang, Cheng-Shium, Havens y Chesney (2003) observaron una alta tasa de enfermedades mentales en las mujeres con VIH: encontraron depresión y SEPT (entre el 50 y 89\%). Otras investigaciones también han mostrado una alta incidencia de depresión en los pacientes con VIH/SIDA, sobre todo entre las mujeres (Jones et al., 2003; Kimmerling, Wagner y Ghosh-Dastidar, 2003), además de altos niveles de tensión psicológica e historias de traumas extremos. Kimmerling, et al. (2003), encontraron una prevalencia importante de depresión en las mujeres VIH positivas y un bajo nivel de sobrevivencia de estas pacientes, lo cual pudiera estar reflejando deficiencias altas en su nivel de adherencia. Por esta razón, resulta imprescindible evaluar el estado de salud mental de los pacientes con $\mathrm{VIH} / \mathrm{SIDA}$, pues puede tener una relación con su adherencia. En este mismo estudio se encontró que el cuidado psiquiátrico tuvo relación con una mejora en la adherencia de hasta un 50\%.

En lo que respecta a los hombres que tienen sexo con hombres, Berg, Mimiaga y Safren (2005), concluyeron que la depresión era el problema más presentado (58.1\%), el síntoma más mencionado (96.3\%) y la depresión mayor se presentó en el $21.4 \%$ de los sujetos.

Murphy, Johnston, Hoffman, Molina y Lu (2003) también asociaron la depresión con una baja adherencia. Los pacientes que se saltan dosis dicen sentirse deprimidos, apesadumbrados, o simplemente olvidarse o dormirse a la hora de su dosis. Por su parte, Vera-Villarroel, Pérez, Moreno y Allende (2004) solo encontraron diferencias estadísticamente significativas en ansiedad y depresión entre los participantes de su estudio, destacándose mayores índices de ansiedad en hombres y mujeres seropositivos/as y 
heterosexuales. En relación con la depresión, encontraron mayores índices en hombres heterosexuales seropositivos.

Con respecto a la relación entre la salud mental, adherencia y las adicciones, varios estudios han encontrado una coincidencia entre la existencia de un trastorno mental y el uso o abuso de alcohol y drogas, factores que en diversos estudios se han relacionado de manera consistente con la baja adherencia (Carrieri, et al., 2003; Gebo, Keruly y Moore, 2003; Witteveen y Ameijden, 2002).

De acuerdo con Mellins, Havens, Mccaskill, Leu, Brudney y Chesney (2002), hay una prevalencia del 50\% de trastornos psiquiátricos entre las mujeres de su muestra (mujeres afroamericanas y latinas en condición de pobreza e infectadas por el VIH/SIDA), porcentaje alarmantemente alto. Igualmente preocupante fue el hecho de que encontraron una prevalencia del $25 \%$ de desórdenes por abuso de sustancias. Ambos factores, tanto los trastornos psiquiátricos como el desorden por abuso de sustancias, se correlacionaron con una baja adherencia. En otro estudio, Mellins et al. (2003), encontraron que casi el 50\% de las mujeres cumplieron con los requisitos de filtraje de un trastorno psiquiátrico y el $25 \%$ el de desorden por abuso de sustancias. La presencia de un trastorno psiquiátrico o el desorden por abuso de sustancias y el estrés debido al rol ejercido como madres, fueron los dos factores que se correlacionaron de manera más fuerte con la baja adherencia al tratamiento antirretroviral. En el estudio de Whetten, Reif, Ostermann, Pence, Swartz, Whetten, Conover, Bouis, Thielman y Eron (2006) los participantes mostraron una disminución estadísticamente significativa en la sintomatología psiquiátrica cuando se logró reducir el uso ilícito de sustancias y alcohol. Además, los sujetos reportaron menos visitas a las salas de emergencias y era más probable que estuvieran recibiendo medicación antirretroviral, además de medicación psicotrópica adecuada.

Brief et al., (2004) encontraron que los individuos VIH positivos con SEPT presentan, por lo general, mayores niveles de estrés emocional y depresión, factores que facilitan la pobre adherencia, al igual que el consumo de alcohol y drogas. La exposición a un trauma puede tener además muchos otros efectos adversos sobre la persona. Se ha encontrado, por ejemplo, que las personas muy estresadas tienen un funcionamiento inmune más pobre, provocando que su conteo de $\mathrm{CD} 4$ disminuya de manera más rápida. Por su parte, Whetten, Leserman, Lowe, Stangl, Thielman, Swartz, Hanisch y Van Scoyoc (2006) identificaron que el 56\% de los pacientes con VIH/SIDA había sido abusado en algún momento de sus vidas y el 30\% experimentó abuso sexual antes de los trece años, sin diferencias de género. Del total de su muestra, $50 \%$ de los participantes experimentó abuso sexual o abuso físico severo.

En un estudio realizado por Hosek, Harper y Domanico (2005), con jóvenes que estaban bajo medicamento antirretroviral, se encontró que el 
66\% había perdido una dosis en la última semana, el 42\% había perdido una dosis el día anterior y el $80 \%$ había perdido una dosis en las últimas dos semanas. Además, se encontró que un rango más elevado de síntomas depresivos y el comienzo de consumo de marihuana a una menor edad predecían niveles más elevados de no adherencia.

El estudio de la adherencia terapéutica entre los pacientes mexicanos es un tema pendiente. De acuerdo con Piña-López (2002), aún cuando la agenda de investigación sobre el tema del VIH/SIDA en México abarca casi la totalidad de las áreas conocidas, el problema de la adherencia terapéutica en cierto modo ha pasado desapercibida para los investigadores de nuestro país.

Estudios realizados con población latina en los Estados Unidos muestran lo siguiente. Murphy et al., (2004) realizaron un estudio que identificó que sólo un 32\% de hablantes monolingües del español mostraba una adherencia de 100\% al HAART cuando se hacía la comparación entre el régimen prescrito y los autorreportes (ayer, antier y el domingo anterior). En otra investigación Power, Koopman, Volk, Ski, Stone, Chesney y Spiegel (2003), encontraron que las personas que se identificaban como heterosexuales y aquellos con raíz latina eran los que significativamente tendían más a ignorar la medicación antirretroviral en los cuatro días anteriores.

Es importante tomar en cuenta la salud mental porque en ocasiones puede poner en riesgo la vida del paciente de manera directa o indirecta. El paciente no aplica el mismo grado de autocuidado personal cuando presenta una enfermedad mental, pues en este caso podría no prestar a la enfermedad del VIH/SIDA la atención que requiere. Pero también, la misma enfermedad mental puede llevar al paciente a terminar de manera involuntaria con su vida, al no tratarse de manera adecuada. Al no adherirse, termina con su vida aunque de una manera más lenta y progresiva, aunque también pueden presentarse riegos de suicidio. El estudio de Schwartz, Wilson, Holman, Weber, Cook y Minkoff, (2006), mostró una prevalencia de ideas suicidas entre los pacientes con VIH/SIDA del $8 \%$, lo cual debería motivar a ofrecerles un fácil y adecuado acceso a la atención psicológica y psiquiátrica que requieren.

Tomando en cuenta los resultados reseñados hasta ahora, la principal pregunta de investigación del presente trabajo es ¿cuál es la relación entre la adherencia terapéutica y algunos indicadores de enfermedad mental en pacientes mexicanos infectados por VIH/SIDA? Otras preguntas de investigación que orientan el presente trabajo son: ¿cuál es la relación entre la adherencia terapéutica y algunas variables sociodemográficas (sexo, escolaridad, empleo), en pacientes mexicanos infectados por VIH/SIDA?, y ¿cuál es la relación entre la adherencia terapéutica y algunas situaciones de vida que enfrentan estos pacientes (apoyo familiar, relación con el personal 
médico, confidencialidad)? Las respuestas a estas preguntas son vitales para diseñar estrategias de intervención más integrales que mejoren la adherencia de los pacientes y con esto aumenten su calidad de vida y sus posibilidades de supervivencia.

\section{Método}

El objetivo principal de este estudio es identificar indicadores de enfermedad mental en una muestra de pacientes mexicanos con VIH/SIDA y definir su relación con la adherencia terapéutica. También se busca establecer la relación entre la adherencia terapéutica y algunas variables sociodemográficas y ciertas situaciones de vida de estos sujetos.

\section{Participantes}

Se realizaron entrevistas a profundidad con pacientes infectados con VIH/SIDA. Los pacientes fueron asignados a los investigadores por los médicos infectólogos tratantes de un hospital público del estado de San Luis Potosí, México, de tal suerte que no hubo un proceso intencionado de selección. Los sujetos residen en su mayoría en zonas suburbanas o rurales. Algunos de ellos fueron referidos al estudio expresamente por contar con una muy buena o muy mala adherencia. En algunos casos, también refirieron a los pacientes para que recibieran apoyo psicológico o bien para motivarlos a iniciar o mantener una adherencia terapéutica alta. A todos los participantes se les informó sobre los propósitos de la investigación y se obtuvo su consentimiento verbal para trabajar con los datos, respetando su confidencialidad.

En el contexto del presente trabajo, se utilizaron los criterios del DSMIV para la identificación de los indicadores de enfermedades o trastornos mentales. Podemos definir la enfermedad mental como el funcionamiento inapropiado de pensamientos, sentimientos, estados de ánimo, capacidad para relacionarse con otras personas o pérdida de la capacidad de actuar autónomamente. La enfermedad mental impide a la persona que la padece interactuar normalmente con el medio en el que vive (NAMI, s/f). La enfermedad mental se identifica por sus manifestaciones: signos (manifestación objetiva de un estado patológico, los signos son observados por el clínico, más que descritos por el individuo afectado) y síntomas (manifestación subjetiva de un estado patológico). Los síntomas son descritos por el individuo afectado, más que observados por el examinador (Psicomed, s/f). El conjunto de signos y síntomas conforman los indicadores de enfermedad mental, los cuales se determinan mediante la entrevista y la observación. 
Por otro lado hay distintas manera de definir la adherencia terapéutica. En la presente investigación se trabajó con el concepto de adherencia terapéutica propuesto por la OMS: el grado en el cual el comportamiento del paciente -la toma de los medicamentos y la introducción de cambios en su estilo de vida- responde a las indicaciones o recomendaciones dadas por el profesional de la salud. En esta definición se destaca la importancia del acuerdo y la participación del paciente con su tratamiento y, por tanto, como un agente activo que colabora con los profesionales de la salud en su propia atención (OMS, 2004). La adherencia, entonces, es un concepto complejo, producto de la interacción de distintas variables. Se tomaron en cuenta los lineamientos proporcionados por Ingersoll (2004) para su valoración: preguntar a los pacientes por los medicamentos que se tomaron la semana anterior, preguntar si se han quedado alguna vez sin sus medicamentos, y cuestionar a los pacientes si siempre toman todos sus medicamentos como se les han prescrito.

En este trabajo se presentan los datos de 76 participantes $(29$ mujeres y 47 hombres). Como se puede apreciar en la Tabla 1, la media de edad de los hombres fue de 32 años y 31 años para las mujeres, la escolaridad promedio fue de seis años (escuela primaria). El 16\% de la muestra asumió una orientación homosexual (todos hombres). Un poco más de la mitad de los sujetos cursó estudios hasta primaria y cerca del $80 \%$ de la muestra cursó estudios hasta secundaria. El 45\% de la muestra permanece soltero y las siete personas viudas son mujeres. Todas las mujeres se contagiaron por su pareja heterosexual y el $20 \%$ de la muestra por vía homosexual. A continuación en la Tabla 1, se presentan los principales datos demográficos.

\section{Instrumentos}

Todas las personas participantes respondieron a una entrevista en profundidad (técnica frecuentemente utilizada en este tipo de investigaciones, por ejemplo Campero, Herrera y Kendall, 2006; Infante, Zarco, Cuadra, Morrison, Caballero, Bronfman y Magis, 2006), aplicada por los investigadores, quienes no se encontraban involucrados con los servicios médicos prestados al paciente. La entrevista estuvo formada por una mayoría de preguntas abiertas para indagar sobre los siguientes temas: datos sociodemográficos, nivel de adherencia, estrategias de adherencia, nivel de apoyo familiar, confidencialidad, información de su estatus a amigos, familiares y parejas, entre otros. Es importante mencionar que a todos los participantes entrevistados se les ofreció apoyo psicológico en caso de considerarlo necesario. 
Ismael García, Fabiola Alfaro, Mónica Rodríguezy Omar Sánchez.

Tabla 1.

Características sociodemográficas de los participantes

\begin{tabular}{lcc}
\hline \multicolumn{1}{c}{ Variable } & Frecuencia & Porcentaje \\
\hline Género & & \\
Masculino & 47 & 62 \\
Femenino & 29 & 38 \\
& & \\
Preferencia sexual & 64 & 84 \\
Heterosexual & 12 & 16 \\
Homosexual & & \\
& & \\
Escolaridad & 41 & 54 \\
Primaria & 18 & 24 \\
Secundaria & 7 & 9 \\
Preparatoria & 10 & 13 \\
Profesional & & \\
& & \\
Estado civil & 34 & 45 \\
Soltero & 29 & 38 \\
Casado & 1 & 1 \\
Divorciado & 7 & 9 \\
Viudo & 5 & 7 \\
Unión libre & & \\
Modo de transmisión & & \\
Vía sexual & 52 & 68 \\
Vía homosexual & 15 & 20 \\
Uso compartido de agujas & 2 & 9 \\
No sabe & 7 & \\
\hline
\end{tabular}

\section{Procedimiento}

Se trabajó en un hospital público ubicado en San Luis Potosí, en la región central de México. En el período que duró la investigación, los pacientes eran atendidos exclusivamente en este lugar, la mayoría provenía de zonas rurales, por lo que les tomaba varias horas llegar al hospital, así que se aprovechó el día de sus consultas médicas para realizar las entrevistas.

Además de la entrevista mencionada en el apartado anterior, se hacía un examen mental para identificar indicadores de enfermedad mental (signos y síntomas). Inmediatamente después de cada entrevista (realizada en todos los casos por dos de los investigadores), se contrastaban los indicadores registrados por cada investigador y luego, en caso de acuerdo, se categorizaban en uno de los síndromes definidos en el DSM-IV. 
Análisis de los datos. Se analizaron los datos mediante estadísticos descriptivos y se compararon mediante las técnicas Chi cuadrada y análisis de correlaciones. En primer lugar, se determinaron los porcentajes de pacientes con indicadores de enfermedad mental, sus correspondientes niveles de adherencia y su relación con algunas variables sociodemográficas. Posteriormente, se analizaron los datos con la Chi cuadrada con el fin de identificar si los grupos conformados con base en el género, la edad, el estado civil, la educación, el nivel de adherencia, las estrategias de adherencia, los indicadores de enfermedad mental, el apoyo social y familiar, la práctica de relaciones sexuales actuales, la relación con el personal médico y la preocupación por la confidencialidad, mostraban diferencias estadísticamente significativas. Por otro lado, se buscó identificar la fuerza de la relación entre la variable escolaridad con las demás, pues se pensó que esta variable podría explicar algunos de los resultados del análisis descriptivo.

Adherencia al tratamiento. La adherencia al tratamiento fue medida a través del auto-reporte de los pacientes y, en la mayoría de los casos, se obtuvo la información de los niveles de CD4 y de su carga viral. Hubo necesidad de recurrir al médico o al expediente porque por lo general, los pacientes desconocían sus conteos o no les interesaba siquiera conocerlos, por no entender su significado ni su trascendencia. Además, se indagaron las estrategias que el paciente ponía en práctica para el mantenimiento de la adherencia, como son: traer las pastillas consigo siempre, colocarlas en un lugar visible, recordatorio con ayuda de alguien o con apoyo familiar o social, también se cuestionó con respecto a la principal motivación para mantenerse adherentes. El nivel de adherencia se clasificó de acuerdo con el número total de pastillas perdidas en el último mes, considerándose excelente $(95 \%$ o más de adherencia); bueno (85\% a $95 \%)$; regular $(60 \%$ a $85 \%$ ) y malo (menos del 60\%), clasificaciones confirmadas por sus conteos y, sobre todo, por su porcentaje de asistencia a consulta. Este porcentaje es particularmente importante porque los médicos prescriben la cantidad exacta de medicamentos, de tal forma que si un paciente pierde una consulta seguramente se quedará sin medicina por espacio de uno o dos meses, tiempo en que el sistema de salud otorga una nueva cita. Se tomaron en cuenta para esta clasificación las ideas de Remor (2002), Hernández y Matos (2008) y Tafur-Valderrama, Ortiz, Alfaro, García-Jiménez y Faus (2008).

\section{Resultados y discusión}

El $82 \%$ de los pacientes cumple con alguno de los indicadores de enfermedad mental: $28 \%$ presenta indicadores de conducta antisocial (como 
uso de drogas o abuso de alcohol, tendencia a la manipulación), 20\% presenta depresión clínica (manifestada como aplanamiento afectivo, desinterés por su entorno y por sí mismos, pesimismo y pensamientos de muerte, principalmente), 13\% síndrome de estrés postraumático (insomnio, con imágenes recurrentes de muerte o del evento en que se infectaron, ansiedad generalizada, alerta incrementada), $12 \%$ psicosis orgánica (alucinaciones auditivas y visuales, pensamientos delirantes) y $8 \%$ indicadores de discapacidad intelectual (desconocimiento de información básica como estaciones del año, nombre del presidente, capital del estado; desinterés por una vida en condiciones de pobreza extrema; ignorancia casi absoluta con respecto a su enfermedad). Aunque el porcentaje de pacientes infectados con $\mathrm{VIH} / \mathrm{SIDA}$ que cumple con alguno de los indicadores de enfermedad mental parece muy alto $(82 \%)$, concuerda con los resultados de algunas investigaciones (Wagner, et al., 2004; Ingersoll, 2004; Murphy, et al., 2004; Delahanty, et al., 2004; Brief, et al., 2004; Carvalho y Leal, 1990, por mencionar sólo algunos).

Se esperaría, al igual que las conclusiones de Mellins, et al. (2003) y de Murphy, et al. (2003), que los pacientes sin indicadores de enfermedad mental mostraran la mayor adherencia. Sin embargo, del total de la muestra solamente cinco pacientes alcanzan un excelente nivel de adherencia $\mathrm{y}$, de ellos, tres presentan indicadores de conducta antisocial, uno de depresión y solamente uno sin indicadores de enfermedad mental. Estos datos se complican si se considera que, comparados con pacientes con los mismos indicadores de enfermedad mental, $47 \%$ de los pacientes con depresión muestra un nivel de adherencia malo o muy malo, lo mismo que $52 \%$ de los pacientes con conducta antisocial, $90 \%$ de los que presentan el síndrome de estrés postraumático y el $100 \%$ de los que presentan discapacidad intelectual (ver Tabla 2). Tal como menciona Arendt (2006), es difícil saber si la relación tan compleja entre los indicadores de enfermedad mental y la adherencia puede deberse a la preexistencia, en muchos casos, de estos indicadores, y el hecho de que la enfermedad mental facilite la realización de conductas de riesgo y el contagio.

Es claro que los indicadores de enfermedad mental no mantienen una relación lineal con la adherencia terapéutica y es altamente probable que interactúen de manera decisiva con variables como el apoyo familiar, condiciones personales de vida, acceso al sistema de salud, condiciones de confidencialidad y otras.

La chi cuadrada mostró diferencias significativas entre las siguientes variables:

Género: con preferencia sexual (chi $=8.8,1 \mathrm{gl}, p=.002 ; 30 \%$ de la muestra se asumió como homosexual, en todos los casos hombres); estado civil (chi $=15.61 \mathrm{gl}, p=.003$ ), la mayor parte de los hombres son casados o solteros, mientras que las mujeres son casadas, solteras, viven en unión libre 
o son viudas. En cuanto al modo de transmisión (chi $=17.3,3 g l, p=.001$ ), un tercio de los hombres se contagió por relaciones homosexuales y dos participantes por compartir agujas, mientras que todas las mujeres se contagiaron por vía heterosexual. Relaciones sexuales (chi $=6.6,2 \mathrm{gl}, p=.03$ ), los hombres mantienen una mayor actividad sexual.

Tabla 2.

Nivel de adherencia, estrategias de adherencia, apoyo familiar, indicadores de salud mental y preocupación por la confidencialidad.

\begin{tabular}{lcc}
\hline \multicolumn{1}{c}{ Variable } & Frecuencia & Porcentaje \\
\hline Nivel de adherencia & 5 & 7 \\
Excelente & 25 & 33 \\
Buena & 20 & 26 \\
Regular & 26 & 34 \\
Mala & & \\
& & \\
Estrategias de adherencia & 7 & 9 \\
Traerlas siempre & 12 & 15 \\
Tenerlas en un lugar visible & 9 & 11 \\
Recordatorio de alguien & 48 & 63 \\
Ninguna & & \\
& & \\
Apoyo familiar & 24 & 31 \\
Ninguno & 12 & 16 \\
Poco & 19 & 25 \\
Regular & 18 & 24 \\
Bueno & 3 & 4 \\
Muy bueno & & \\
& & \\
Diagnóstico de salud mental & 14 & 18 \\
Sin indicadores de enfermedad mental & 15 & 20 \\
Depresión & 10 & 13 \\
Síndrome de estrés postraumático & 21 & 28 \\
Conducta antisocial & 10 & 13 \\
Psicosis & 6 & 8 \\
Discapacidad intelectual & & \\
Preocupación por la confidencialidad & 71 & 93 \\
Sí & 5 & 7 \\
$\quad$ No & & \\
\end{tabular}


Escolaridad: empleo (chi $=44.5,12 \mathrm{gl}, p=.000$ ) y nivel de adherencia (chi $=11.7,9 \mathrm{gl}, \mathrm{p}=.011$ ). Esta variable es muy importante, pues muestra que la escolaridad sí tiene un efecto importante sobre la adherencia terapéutica (y sobre el empleo). Las personas con mayor escolaridad muestran una mayor adherencia porque se interesan más por conocer las características de la enfermedad y su curso.

Nivel de adherencia: estrategias de adherencia (chi= 36.8, $12 \mathrm{gl}, p=$ .000 ), apoyo familiar (chi $=21.6,12 \mathrm{gl}, p=.01$ ) y confidencialidad (chi $=4.6,3$ $g l, p=.03)$. Estos resultados también resultan particularmente interesantes, pues indican que para el logro de la adherencia terapéutica hace falta seguir una estrategia especial, como tener las medicinas siempre en un lugar visible o traerlas todo el tiempo y también establece que el apoyo familiar es vital para la adherencia. Al analizar de una manera más cualitativa este último dato, se encontró que los hombres que tienen apoyo familiar son más adherentes. Las mujeres cuentan con menor apoyo familiar. Sin embargo, muestran un alto nivel de adherencia cuando tienen bajo su cuidado a los hijos o al esposo.

Con respecto a las correlaciones, éstas fueron significativas entre escolaridad y nivel de adherencia $(r=.297, p=.005)$, resultado que concuerda con el presentado antes. Otra correlación significativa se encontró entre la escolaridad y la relación con el personal médico $(\mathrm{r}=.207, p=.03)$. Este último resultado podría indicar que, a mayor escolaridad se produce una mayor interacción entre los participantes y sus médicos. Las personas con menor escolaridad generalmente asumen una actitud muy pasiva frente a sus médicos y no preguntan, no indagan, no cuestionan y no exigen información o explicaciones acerca de su tratamiento. Como comentan Johnston y Mann (2003), algunos pacientes se sienten muy angustiados al tener que decir al personal médico acerca de sus problemas de adherencia, lo que debe alertar a dicho personal a ser sensible, que entienda lo difícil que es su logro. Ramírez, Palacios-Martínez y Guerra (1993) mencionan que es frecuente encontrar profesionales de la medicina que prescinden de una actitud de empatía y por lo tanto impiden la co-atención de los pacientes, recurriendo al psicólogo sólo ante situaciones de angustia intensa en los consultantes, limitando con esta actitud el logro de una buena salud física y emocional del paciente.

Tomando en cuenta lo anterior, se considera que los pacientes con $\mathrm{VIH} / \mathrm{SIDA}$ tienen derecho y deben tener acceso a las facilidades médicas y a los otros tratamientos que les permitan sobreponerse más rápidamente al diagnóstico y, con ello, aumentar su nivel de adherencia terapéutica, por lo que, como dicen Johnston y Mann (2003), los servicios de psicología y psiquiatría deberían estar siempre disponibles.

Hay estudios que muestran claramente los beneficios del tratamiento dado a los pacientes con enfermedad mental y VIH, por ejemplo el realizado por Whetten, Reif et al., (2006), quienes observaron cómo después del 
tratamiento, los pacientes mostraron una disminución en su sintomatología psiquiátrica, además tuvieron una disminución en el uso de sustancias y alcohol. Otro ejemplo es el de Jones et al., (2003), quienes ofrecieron terapia a 174 mujeres con SIDA, la cual consistió en diez sesiones de terapia cognitivo-conductual/terapia de apoyo expresivo. Se formaron dos grupos, un grupo control y uno experimental. Al igual que en el presente estudio encontraron una correlación alta entre el nivel de conocimientos acerca del VIH y de la medicación con el nivel de adherencia.

Por su parte, Kimmerling, et al. (2003) encontraron que en hombres y mujeres con depresión, el cuidado psiquiátrico por sí mismo estaba relacionado con un $50 \%$ de mejora en la adherencia, mientras que la terapia antidepresiva por sí sola mostraba efectos positivos, pero no significativos. De hecho, la mayoría de las personas depresivas preferían la consejería a la terapia farmacológica.

Por otro lado, el hecho de que la mayoría de la muestra del presente estudio viva en lugares semiurbanos o rurales puede ser importante, pues Reif, Whetten, Ostermann y Raper (2006) observaron que los participantes que vivían en zonas más rurales tendían a reportar con menor frecuencia visitas a un proveedor de la salud mental. Los participantes que vivían en zonas más rurales, al ser comparados con los urbanos, poseían niveles más altos de malestar psicológico, aunque éste no mostró ser estadísticamente significativo. En el caso del presente estudio, muchos pacientes viven en zonas rurales, alejadas del centro hospitalario donde reciben atención, lo que sin duda contribuye a una adherencia pobre.

\section{Conclusiones}

Solamente el $20 \%$ de la muestra de este estudio alcanzó un nivel de adherencia terapéutica apropiado, resultado a todas luces insatisfactorio. Se encontró que a menor escolaridad, menor adherencia terapéutica (la escolaridad de la muestra estudiada fue en general baja). También se encontró que, a mayor conocimiento de la enfermedad corresponde una mayor adherencia. Esto es muy importante porque implica que deben realizarse campañas más enfocadas a proporcionar información puntual, objetiva y clara a los pacientes infectados con VIH/SIDA, a fin de aumentar su nivel de adherencia.

Es probable que el personal médico de una institución pública de salud, generalmente sobrecargado por el exceso de trabajo, no sea el indicado para proporcionar mayor información a los pacientes, pues las personas con una formación escolar pobre probablemente se sienten intimidados o incómodos en su presencia, y entonces no van a cuestionar ni a plantear sus dudas o 
reticencias con respecto al tratamiento. Es razonable pensar que los psicólogos pueden desempeñar esta función con mayor atingencia.

Otro resultado importante es la relación entre la adherencia terapéutica y el apoyo familiar. Este apoyo se complica porque a menudo los pacientes con VIH/SIDA tienen una gran preocupación por la confidencialidad. Estos pacientes necesitan saber que comentar su estatus con sus familiares es muy importante para su bienestar.

Los pacientes también deben saber que es vital que desarrollen una estrategia para evitar los olvidos en la toma de sus medicamentos. A muchos pacientes les funciona muy bien tener sus pastillas en lugares visibles de la casa, traerlas siempre consigo o bien pedir el apoyo de un familiar para recordarles.

Un alto porcentaje de los participantes en este estudio mostró algún indicador de enfermedad mental $(80 \%)$, y aunque no se encontró una relación determinante entre esta condición y la adherencia terapéutica, es un hecho que debe ofrecerse a estas personas servicios de salud mental.

$\mathrm{La}$ adherencia terapéutica depende de varios factores o de varias combinaciones de factores. Se piensa que uno de ellos es la presencia de indicadores de enfermedad mental, pero otros como el apoyo familiar y social, la escolaridad, el conocimiento de la enfermedad, el tiempo que se ha vivido con el virus, las facilidades de acceso al sistema de salud, la situación económica y constructos personales en relación con la enfermedad pueden ser también determinantes, por lo que se necesitan investigaciones que aborden estas combinaciones para definir su impacto en la población de pacientes afectados por el VIH/SIDA.

\section{Referencias}

Arendt, G. (2006). Affective Disorders in Patients with HIV Infection Impact of Antiretroviral Therapy. CNS Drugs, 20(6), 507-518.

Balfour, L., Silvermani, K., Tasca, G. A., Angelli, J. B., Macpherson, P. A., Garber, G., Cooper, C. L. \& Cameron, D. W. (2006). A randomized controlled psycho-education intervention trial: Improving psychological readiness for successful HIV medication adherence and reducing depression before initiating HAART. AIDS Care, October; 18(7): 830-838

Barrios-Nuñez, J., Solís-Cano, F.J. \& Feregrino, M. (2006). Prevention of suicide for depression in patients with HIV/AIDS (resumen). Ponencia presentada en la XVI International AIDs Conference Abstracts, Toronto, Canadá, CDC 1925. http:/ / bvssida.insp.mx/resultados.php?criterio=barrios-nu $\% \mathrm{~F} 1 \mathrm{ez} \&$ seltabla $=1$, recuperado el 15 de junio de 2008.

Berg, M., Mimiaga, M. \& Safren, S. (2005). Mental Health Concerns of HIV-Infected Gay and Bisexual Men Seeking Mental Health Services: An Observational Study AIDS Patient Care and STDs, 18(11), 635-643.

Brief, D., Bollinger, A., Vielhauer, M., Berger-Greenstein, J., Morgan, E., Brady, S., Buondonno, L. \& Keane, T. (2004). Understanding the interface of HIV, trauma, post- 
traumatic stress disorder, and substance use and its implications for health outcomes. AIDS Care, 16(Suppl. 1), 97-120.

Broyles, A. \& Erlen, J. (2005). Medication Practice and Feminist Thought: A theoretical and Ethical Response to Adherence in HIV/AIDS. Bioethics, 19(4), 362-378.

Carrieri, M., Chesney, M., Spire, B., Loundou, A., Sobel, A., Lepeu, G., Moatti, J., \& MANIF Study Group (2003). Failure to Maintain Adherence to HAART in a Cohort of French HIV-Positive Injecting Drug Users. International Journal of Behavioral Medicine, 10(1), 1-14.

Campero, L., Herrera, C. \& Kendall, T. (2006). Adherente treatment related to the doctorpatient relationship: a qualitative study in Mexico. XVI International Aids Conference Abstracts. TUPE 0819

Carrillo-Martínez H., García-Lozada J. \& Sierra-Corona B. (2005). Estrategias para mejorar la adherencia al tratamiento antirretroviral (resumen). Ponencia presentada en el $9^{\circ}$. Congreso Nacional sobre VIH/SIDA e Infecciones de Transmisión Sexual, Oaxaca, México, No. 4104. Recuperado el 15 de julio de 2008 de: http:/ / bvssida.insp.mx/harticulo.php?id_art=4104\&seltabla=1

Carvalho J. A. \& Leal, I. (1990). Psicología de saúde. Contexto e intervenção. Análise Psicológica, VIII, 453-458.

Chesney, M. (2003). Adherence to HAART Regimens. AIDS Patient Care and STDs, 17(4), 169175.

Ciasullo, E. \& Escovitz, K. (2005). Positive futures: The need for paradigm shift in HIV/AIDS services. Journal of Vocational Rehabilitation 22, 125-128.

Delahanty, D. L., Bogart, L. M. \& Figler, J. L. (2004). Posttraumatic stress disorder symptoms, salivary cortisol, medication adherence and CD4 levels in HIV-positive individuals. AIDS Care 16(2), 247-260.

Demmer, C. (2003). Attitudes Toward HIV Proteasa Inhibitors and Medication Adherence in an Inner City HIV Population. Aids Patient Care and STDs, 17 (11), pp. 575-580

Gebo, K., Keruly, J. \& Moore, R. (2003). Association of social stress, Illicit Drug Use, and Health Beliefs with Nonadherence to Antiretroviral Therapy. J. Gen Intern Med, 18, 104111.

Hernández, D. \& Matos, D. (2008). Reglamentación técnica para mejorar la adherencia al tratamiento antiretroviral en Venezuela. Programa Nacional de SIDA ITS. Gobierno Bolivariano de Venezuela.

Hill, Z., Kendall, C. \& Fernández, M. (2003). Patterns of Adherence to Antiretrovirals: Why Adherence Has no Simple Measure. Aids Patient Care Ans STDs, 17 (10), pp. 519-525.

Horne, R., Buick, D., Fisher, M., Leake, H., Cooper, V. \& Weinman, J. (2004). Doubts about necessity and concerns about adverse effects: identifying the types of beliefs that are associated with non-adherence to HAART. International Journal of STD \& AIDS, 15, 3844

Ingersoll, K. (2004). The impact of psychiatric symptoms, drug use, and medication regime on non-adherence to HIV treatment. AIDs Care, 16(2), 199-211.

Infante, C., Zarco, A., Cuadra, S. M., Morrison, K., Caballero, M., Bronfman, M. \& Magis, C. (2006). El estigma asociado al VIH/SIDA: el caso de los prestadores de servicios de salud en México. Salud Pública de México , 48 (2), marzo-abril.

Johnson, M., Chesney, M., Goldstein, R., Remien, R., Catz, S., Gore-Felton, C., Charlebois, E. \& Morin, S. (2006). Positive Provider Interactions, Adherence Self-Efficacy, and Adherence to Antiretroviral Medications Among HIV-Infected Adults: A Mediation Model. Aids Patient Care and STDs., 20(4), pp. 258-268

Jones, D. L., Ishii, M., LaPerriere, A., Stanley, H., Antoni, M., Ironson, G., Schneiderman, N., Van Splunteren, A., Cassells, A., Alexander, K., Gousse, Y. P., Vaughn, A., Brondolo, E., Tobin, J. N. \& Weiss, S. M. (2003). Influencing Medication Adherente Among Women with AIDS. AIDS Care,15(4), 463-474. 
Johnston, K. \& Mann, T. (2003). Adherence to Antiretroviral Medications in HIV/AIDS Care: a Narrative Exploration of One Woman's Foray into Intentional Nonadherence. Health care for Women International, 24, 552-564.

Kimmerling, M., Wagner, G. \& Ghosh-Dastidar, B. (2003). Relationship of Gender, Depression and Health Care Delivery with Antiretroviral Adherence in HIV Infected Drug Users. International Journal of STD \& AIDS, 14, 281-284

López-Martínez, C., Ortíz-Mondragón, R., Moreno-Monroy, T., Sierra-Corona, B.,MoralesCarcaño, A, Magis-Rodríguez, C., Hernández-Tepichín, G. \& Baez-Villaseñor, J. (1999). Evaluación cualitativa de la adherencia terapéutica en pacientes infectados con VIH (resumen). Ponencia presentada en el VI Congreso Nacional Sobre SIDA. Honduras. Recuperado el 24 de noviembre de 2008 de: http://bvssida.insp.mx/harticulo.php?id_art $=2403 \&$ seltabla $=1$

Mellins, C., Havens, J., Mccaskill, E., Leu, C., Brudney K., \& Chesney, M. (2002). Mental health, substance use and disclosure are significantly associated with the medical treatment adherence of HIV-infected mothers Psychology, Health \& Medicine, 7(4), 451460.

Mellins, C., Kang, E., Cheng-Shiun, L., Havens, J. \& Chesney, M. (2003). Longitudinal Study of Mental Health and Psychosocial Predictors of Medical Treatment Adherence in Mothers Living with HIV Disease. AIDS Patient Care and STDs, 17(8), 407-416.

Murphy, D. A., Marelich, W. D., Hoffman, D. \& Steers, W. N. (2004). Predictors of antiretroviral adherence. AIDs Care, 16(4), pp. 471-484.

Murphy, D., Johnston, K., Hoffman, D., Molina, A. \& Lu, C. (2003). Barriers and successful strategies to antiretroviral adherence among HIV infected monolingual Spanishspeaking patients. AIDS Care, 15(2), 217-230.

National Alliance of Mental Illness ( $/ \mathrm{f})$. What is mental illness? Mental illness facts Recuperado el 26 de junio de 2010 de: http://www.nami.org/Content/NavigationMenu/Inform_Yourself/About_Mental_Illn ess/About_Mental_Illness.htm

Organización Mundial de la Salud. (2004). Adherencia a los tratamientos a largo plazo: pruebas para la acción. Recuperado el 26 de junio de 2006 de: http://www.paho.org/Spanish/AD/PC/NC/nc-adherencia.htm

Paredes-Castellanos E. (2002). La carta compromiso como instrumento para la adherencia terapéutica (resumen). Ponencia presentada en el VIII Congreso Nacional Sobre SIDA e Infecciones de Trasmisión Sexual. Veracruz, México. Recuperado el 6 de enero de 2009, de: http://bvssida.insp.mx/harticulo.php?id_art=3416\&seltabla=1.

Piña-López J A. (2002). Adherencia terapéutica y VIH/SIDA: una relación ausente en la agenda de investigación en México (resumen). Ponencia presentada en el VIII Congreso Nacional Sobre SIDA e Infecciones de Trasmisión Sexual. Veracruz, México. Recuperado el 6 de enero de 2009, de: http://bvssida.insp.mx/harticulo.php?id_art=3557\&seltabla=1,

Power, R., Koopman, C., Volk, J., Ski, D., Stone, L., Chesney, M. \& Spiegel, D. (2003). Social Support, Substance Use, and Denial in Relationship to Antiretroviral Treatment Adherence among HIV-Infected Persons AIDS Patient Care and STDs, 17(5), 245-252.

Psicomed (s/f). Glosario de términos técnicos. Recuperado el 26 de junio de 2010, de: http://personal.telefonica.terra.es/web/psico/dsmiv/dsmiv_glosario.html

Ramírez, A., Palacios-Martínez, M. \& Guerra, A. (1993). La importancia del trabajo psicológico en la adherencia a tratamientos médicos en personas que viven con VIH y SIDA (resumen). Ponencia presentada en el IV Congreso Nacional Sobre SIDA. Recuperado el 6 de enero de 2009, de: http:// bussida.insp.mx / harticulo.php?id_art $=353$ \&seltabla $=1$ 
Reback, C. J., Larkins, S. \& Shoptaw, S. (2003). Methamphetamine abuse as a barrier to HIV medication adherence among gay and bisexual men. AIDs Care, 15(6), 775-785.

Reif, S., Whettan, K., Ostermann, J. \& Raper, J. (2006). Characteristics of HIV-infected adults in the Deep South and their utilization of mental health services: A rural vs. urban comparison. AIDS Care, 18(Suppl.1), 10-17.

Remor, E. (2002). Valoración de la adhesión al tratamiento antirretroviral en pacientes VIH+. Psicothema, 14(2), 262-267.

Royal, S., Cohn, S., Kwait, J. Kidder, D., Wolitski, R., Stall, R., Aidala, A. \& Holtgrave, D. (2006). Factors associated with adherence to HIV medications in homeless or unstably housed persons living with HIV (resumen). Ponencia presentada en la XVI International Aids Conference Abstracts, Toronto, Canadá. Abstract no. TUPE0150. Recuperado el 16 de junio de 2009, de: http:/ / www.iasociety.org/Default.aspx?pageId=11\&abstractId=2191344,

Schwartz, R., Wilson, T., Holman, S., Weber, K., Cook, J. \& Minkoff, H. (2006). Associations between HIV status, aging and mental health among U.S. urban, minority woman (resumen). Ponencia presentada en la XVI International AIDs Conference Abstracts, Toronto, Canadá. Abstract no. THPE0612. Recuperado el 16 de junio de 2009, de: http:/ / www.iasociety.org/Default.aspx?pageId=11\&abstractId=2195247

Tafur-Valderrama, E., Ortiz, C., Alfaro, C. O., García-Jiménez, E. \& Faus, M. J. (2008). Adaptación del "Cuestionario de Evaluación de la Adhesión al Tratamiento antirretroviral” (CEAT-VIH) para su uso en Perú. Ars Pharm; 49 (3), 183-198.

Torres-Escobar, I, González-López, C \& Martínez-Fernández, R. (2002). La adherencia en mujeres rurales mexicanas (resumen). Ponencia presentada en la VIII Congreso Nacional Sobre SID A e Infecciones de Trasmisión Sexual. Veracruz, México. Recuperado el 4 de enero de 2009, de: .http://bvssida.insp.mx/harticulo.php?id_art=3491\&seltabla=1

Uldall, K., Palmer, N., Whetten, K. \& Mellins, C. (2005). Adherence in people living with HIV/AIDS, mental illness, and chemical dependency: a review of the literature. AIDS Care, 16(Suppl.1), 71-96.

Vera-Villarroel, P., Pérez, V., Moreno, E. \& Allende, F. (2004). Diferencias en variables psicosociales en sujetos VIH homosexuales y heterosexuales. International Journal of Clinical and Health Psychology, 4(1), 55-67.

Wagner, G., Kanouse, D., Koegel, P. \& Sullivan, G. (2003). Adherence to HIV Antiretrovirals among Persons with Serious Mental Illness. AIDS Patient Care and STDs, 17(4), 179-186.

Wagner, G. J., Kanouse, D. E., Koegel, P. \& Sullivan, G. (2004). Correlates of HIV antiretroviral adherence in persons with serious mental illness. AIDs Care, 16(4), 501506.

Weiss, L., French, T., Finkelstein, R., Waters, M., Mukherjee, R. \& Agins, B. (2003). HIVrelated knowledge and adherence to HAART. AIDs Care, 15(5), 673-679.

Whetten, K., Reif, S., Ostermann, J., Pence, B., Swartz, M., Whetten, R., Conover, C., Bouis, S., Thielman, N. \& Eron, J. (2006). Improving health outcomes among individuals with HIV, mental illness, and substance use disorders in the Southeast. AIDS Care, 18(Suppl.1), 18-26.

Whetten, K., Leserman, J., Lowe, K., Stangl, D., Thielman, N., Swartz, M., Hanisch, L. \& Van Scoyoc, L. (2006). Prevalence of Childhood Sexual Abuse and Physical Trauma in an $H I V$-Positive Sample from the Deep South. American Journal of Public Health, 96(6), 10281030.

Witteveen, E. \& Ameijden, E. (2002). Drug Users and HIV-Combination Therapy (HAART): Factors Which Impede or Facilitate Adherence. Substance use and misuse, 37(14), 19051925.

Recibido: 16 de enero de 2009

Aceptado: 30 de junio de 2010 